Gynecol Obstet Invest 1997;43:281-282

\title{
Author Index Vol. 43, 1997
}

Gynecologic and Obstetric Investigation

Ahmed, M.S. 53 Akarsu, C. 55 Akimoto, K. 142 Aksoy, E. 55 Alata§, C. 55

Albunex Study Group 116 Aleman, J. 112 Al-Hendy,A. 268 Altaras, M.M. 60 Anyaegbunam, A. 25 Aoki,S. 155,232 Aono, T. 225 Araki,T. 158 Aravantinos, D. 125 Ariyuki, Y. 232

Athanassiades, P. 125 Athanassiadou, P. 125 Auersperg, N. 268 Azuma, Ch. 166

Bahçeci, M. 55 Barbieri, R.L. 53,276 Barnie-Adshead, A.M. 108 Bengtsson Agostino, M. 84

Berghella,V. 68 Bergmans, M.G.M. 1, 150 Beyth,Y. 60 Bukovsky, I. 178 Burns, P.A. 236

Caffarri,G. 162 Cardamakis, E. 255 Carlström, K. 41 Carrasco, G. 219 Chao,H.-T. 212 Check, J.H. 242 Cheng, X. 195 Cheung, T.H. 200 Chiang, CM. 245 Chiba,H. 171 Chiodera, P. 162

Chung, T.K.H. 200 Cigarini, C. 162 Cohen, I. 60 Coiro,V. 162 Cordoba, M. 60 Cortés-Prieto, J. 192 Cruz,M.A. 219

Degani, S. 131 Diamantis, A. 255 Dietl,J. 261 Dror,Y. 60

Elias,A.N. 47 Ergin,T. 183 Eriksson-Mjöberg, M. 41 Evers,J.L.H. 79

Facchinetti, F. 120 Figer, A. 60 Filmar, S. 73 Francis, M.M. 76 Friedman, Z.M. 11 Fujiwaki, R. 155 Fuke,S. 225 Fukuda-Miki, M. 166

Gadsby,R. 108 Gaffuri,B. 6 Gallardo,V. 219 Genazzani, A. 120 Geppert, M. 261 Gindoff,P.R. 186 Gökmen, O. 183 Gonser,M. 98 Gonzalez, C. 219 Gramellini, D. 162 Grandinetti, G. 120 Gröne,K. 278 Guida,C, 251 Gülekli,B. 183

Haan, J. de 206 Haberman, S. 11 Hadas,E. 178 Halperin,R. 178 Hasaart, T.H.M. 1,150

Hashimoto, K. 166 Hata,K. 135,155,232 Hata,T. 135, 155,232 Hellberg,D. 49 Hill,J.A. 245

Hiroi,M. 171 Hoda,S. 68

Imanaka, M. 64 Inada,K. 155 Inoue,M. 142 Isik,A.Z. 183 Itamochi, H. 195 Itskovitz-Eldor, J. 73 Jadali,D. 25 Jagger,C. 108

Kaiis, M. 34 Railings, I. 49 Kaminski, R. 37 Ranai, T. 166 Rashiwakura, M. 171 Rato,H. 158

Ratz,Z. 204 Rawamura, T. 155 Rawarabayashi, T. 145 Keller, J. 192 Kelly, A.C. 116 Kelly, A.J. 89 Kessels, A.G.H. 79 Reunen,H. 1, 150 Rhong,T.Y. 89 Rielkowski, A. 29 Rigawa,J. 195

Ritao,M. 135,232 Rol,S. 73 Rommoss, F. 278 Rorantzis, A. 255 Rotoulas, I.-G. 255

Lai, Ch.-R. 212 Lam, S.R. 200 Langer, M. 278 Lanzone, A. 251 Lao,T.T. 94 Laubenberger, J. 278 Lee, G.S. 116 Lee,S.S.N. 94 Leszczynska-Gorzelak, B. 37 Leung, J.O. 200 Lightman, A. 73 Liljestrand, J. 112 Lindheim, S.R. 76 Lobo,R.A. 76,215 Lurie, S. 204

Maas,J.W.M. 79 Macaso, T. 76 Madjar, H. 278 Maeda,R. 225 Magotti,M.G. 162 Magri, B. 6 Makihara, R. 232 Mancuso, S. 251 Manor, D. 73 Mantouvalos, C. 255 Mårdh,P.A. 49

Markowitz, J. 34 Marzusch, R. 98 Matsuzaki,N. 225

KAKGER

E-Mail karger@karger.ch Fax+ 41613061234 http://www. karger.ch (C)1997S. KargerAG, Basel

281 
Maymon, R. 178 Metalinos, K. 255 Michalas, S. 125 Michikura,Y. 142 Michopoulos, J. 255 Mielke,G. 98 Miguel, P. 219 Mikhail, M.S. 25 Minagawa, Y. 195 Mitsuda,N. 225 Monaco, A. 251 Mongelli,M. 20 Mori,E. 171 Morioka,Y. 171 Murata, Y. 166 Mushayandebvu, T.I. 209 Nadaoka,T. 171 Nakai,Y. 64 Nakayama, M. 225 Nappi,R.E. 120 Neri,I. 120 Ngadiman, S. 68 Nieto,A. 192 Nikischin, W. 104 Nilsson,S. 49 Nishimura, R. 139 Nishio, J. 64 Nobunaga, T. 166 Odeh,M. 34 Oettinger, M. 34 Ogita, S. 64 Okada,M. 195 Oldigs,H.D. 104 Oleszczuk, J. 37 Ölund,A. 41

Pandian,M.R. 47 Paulson, R.J. 76,215 Peña,R. 112 Persson, L.Å. 112 Pesso,R. 242 Peter, M. 104

Petrakakou, E. 125 Peymer, M. 242 Pietsch-Breitfeld, B. 98

Rabinerson, D. 204 Randolph, G. 25 Regele,B. 98 Relakis,K. 255 Rojas,F.-J. 47 Rosenberg, H. 68 Rossi, G. 6 Rubinow, D.R. 186

Saito,H. 171 Saji,F. 166 Sakellariou, V. 125 Sances,M.G. 120 Sauer,M.V. 76,215 SawulickaOleszczuk, H. 37 Schanler, R.J. 236 Schmidt, P.J. 186 Schneider, B. 278 Schneider, D. 178 Scribanti, A. 251 Senoh,D. 135 Serra,M. 192 Session, D.R. 116 Shapira, J. 60 Shimoya,K. 166,225 Shojo,H. 145 Shozu,M. 142 Sie,G. 206 Sikström,B. 49 Simon, D. 204 Smith, L.G., Jr. 236 Smyczek-Gargya, B. 261 Somigliana, E. 6 Sonoda,Y. 142 Stathopoulos, E. 255 Stein, D. 73 Stevens, G.H. 1, 150 Suehara,N. 225 Sugimura, K. 139 Szymula,D. 37

Tacuri,C. 192 Takemori,M. 139 Tal,J. 131 Tanaka,J. 142 Tee,J.H.-C. 89 Tepper, R. 60 terRiet, G. 79 Terakawa,N. 195 Timor-Tritsch, I. 131 To,K,F. 200 Tokugawa, Y. 166 Tran,T. 25

Tsujimoto, M. 166 Tsukamoto, T. 145 Tzingounis, V. 255

Uszyński, M. 29 Uszyński,W. 29

Vavalà,V. 251 Viganò, P. 6 Vignali, M. 6 Volpi,R. 162 Volz,B. 261

Wall,S. 112 Wang,P.-H. 212 Weiss, G. 209 Wong,Y.F. 200

Yakm,K. 55 Yasuda,D. 139 Yoneyama, Y. 158 Yuan,Ch.-Ch. 212

Zalel,Y. 60 Zekanowska, E. 29 Zerva, Ch. 125 Zorlu,C.G. 183 Zuna, R.E. 68 Zwam, W.H. van 206

282

Gynecol Obstet Invest Vol. 43, 1997

Author Index 